\title{
Dexterous Actuation
}

\author{
M. A. Saliba* and C. Ellul
}

RIAL - Robotics and Industrial Automation Laboratory, Department of Industrial and Manufacturing Engineering, University of Malta, Msida, Malta MSD 2080.

*Corresponding author, michael.saliba@um.edu.mt, tel: +35699458660

\begin{abstract}
Methods that have been developed for actuation system evaluation are normally generic, and primarily intended to facilitate actuator selection. Here, we address specifically those engineering devices that exhibit multiple-degree-of-freedom motions under space and weight constraints, and focus on the evaluation of the total actuation solution. We suggest a new measure that we provisionally call 'Actuation Dexterity', which interrogates the effectiveness of this total solution and serves as a design support tool. The new concept is developed in the context of artificial hands, and the approach is based on the review and analysis of thirty-six different artificial hand projects described in the literature. We have identified forty-eight unique evaluation criteria that are relevant to the actuation of devices of this type, and have devised a scoring method that permits the quantification of the actuation dexterity of a given device. We have tested this approach by evaluating and quantifying the actuation dexterity of five different artificial hands from the literature. Finally, we discuss the implications of this approach to the design process, and the portability of the approach between different device types.
\end{abstract}

Keywords: Actuation, Dexterity, Artificial Hands, MIS Tools

\section{Introduction}

In a review article for the Springer Handbook of Robotics, Melchiorri and Kaneko stated that "one of the main issues [in the mechanical design of an articulated robotic hand] is the design of a proper actuation and transmission system. This aspect is crucial because space and dimensions are usually limited,.." [1].

In a different research field, Kode et al. noted that "developing a device for local actuation is the major challenge in designing new tools for MIS [minimally invasive surgery]" [2].

The above two device types, i.e. dexterous robot hands and articulated tools for minimally invasive surgery, share a common problem - the requirement of an effective actuation system for a multi-degree-of-freedom (MDOF) device that has significant design constraints associated with space and/or weight limitations. The same type of problem can be found in other specialized applications. A third example would be in the design of interplanetary rovers, where MDOF actuation is needed for much of the instrumentation and for the rover 
itself, in a situation where it is crucial to minimize the size and weight of the package due to the constraints imposed by interplanetary delivery.

In this work we examine this general problem of MDOF actuation of dexterous devices in the presence of serious space and weight constraints. As discussed in section 2 below, this requires an evaluation approach that is sufficiently bounded so as to address specifically this class of devices, while at the same time that is sufficiently detailed so as to address all of the subtleties associated with this type of demanding actuation problem.

\section{Background and Proposed Approach}

\subsection{The Evaluation of Actuation Systems}

Various studies on the evaluation of actuation systems can be found in the literature. In general, these are aimed mainly at guiding the engineer in selecting the best actuator for the device / task at hand. A brief overview of some of these studies is given below.

In [3] the authors presented a method for the selection of an actuator for a given task, by matching the actuator performance to the task requirements. They identified nine properties that define the task requirements: force, displacement, stiffness, size, mass, operating frequency, power, efficiency and resolution. They also took note of (but did not analyze) four other requirements: cost, durability, maintenance and environmental impact. The analysis was limited to linear actuators, and the authors identified nine actuator performance characteristics: actuation stress (applied force per unit cross-sectional area of an actuator), maximum actuation stress, actuation strain (ratio of actuator extension to its original length), maximum actuation strain, actuator density (ratio of mass to initial actuator volume), actuator modulus (the ratio of a small increment in actuation stress to the corresponding small increment in actuation strain), volumetric power (mechanical power output per unit initial volume in sustainable cyclic operation), efficiency, and strain resolution.

In [4] the authors developed a database of 220 actuators and implemented the generalized approach suggested in [3]. Apart from the actuator performance characteristics listed above, they also considered six absolute performance characteristics: size, stroke, output force, operating frequency, weight and cost.

In [5] the authors identified three principal actuator attributes: maximum force, frequency range, and stroke; and related the different types of actuators to these attributes. They identified various actuator configurations (e.g. actuators in series) that can reposition an actuator type with respect to one or more of these attributes. They recommended a three step strategy for actuator selection: (i) determine the required force, frequency and stroke; (ii) identify all existing actuator technologies that satisfy the requirements; and (iii) use Pareto analysis to compare candidate technologies, taking into account also any secondary actuator attributes that may be important for the required application. 
In [6] the author has warned that in evaluating different actuators it is important to confirm that the actuator designs are optimized with respect to the critical task requirements. He has taken as an example a comparison between conventional pneumatic actuators and pneumatic artificial muscles (PAMs) on the basis of energy to mass ratios, and has demonstrated that although PAMs come out on top when compared to the commercially available pneumatic actuators, the latter are normally over-designed, and can in fact be refined / customized for specific applications to achieve properties superior to those of PAMs.

A number of studies have been carried out on the evaluation / selection of actuators specifically for use in artificial hands. In [7] the authors compared a number of conventional and non-conventional actuators for use in a prosthetic hand, on the basis of size, weight, flexibility and applicability, precision, hysteresis and non-repeatabilty, energy consumption, operation frequency, efficiency, power density, anthropomorphism, and cost. In [8] the authors evaluated different actuators, also for use in a prosthetic hand, on the basis of efficiency, frequency, energy storage capacity, robustness, specific power, volumetric power, required transmission, self-braking capability, noise, controllability, and safety. In both these studies the selection of attributes to evaluate, as well as the attribute marking schemes, were somewhat arbitrary. Both studies identified brushless DC motors and ultrasonic motors as the most suitable for this application. In [9] the authors compared a number of actuators for use in an artificial hand for a robot mimicking a two-year-old infant, on the basis of various criteria including some brought up in [3] and [4], and selected (brushed) DC motors for their application.

In all of the studies discussed above, the focus has been mainly on the actuator itself, and not on the actuation system as a whole and/or on the general approach and philosophy taken to address the actuation problem. Thus issues such as the location of the actuators, the effectiveness of the transmission system, the synergies with the rest of the device systems, and many of the numerous particularities that pertain to MDOF actuation in constrained environments, were not included in the generalized evaluation paradigms. Furthermore, the existing studies basically serve as actuator selection methods or as measures of actuator suitability for selected applications. The literature is lacking a higher level evaluation protocol that delves into the subtleties of high demand actuation systems, potentially acting as a driver for cutting edge refinements to the system and/or as a trigger for innovative and possibly even radical improvements.

In this work, we suggest an approach to address these gaps. We suggest the introduction of a new niche evaluation framework, which focuses exclusively on the contribution of the actuation system to the overall effectiveness of a dexterous device. This evaluation framework is intended to serve as a comparative and design support tool, and, in the form presented here, to complement, not replace, the traditional actuator evaluation and selection methodologies. We propose a new parameter that we provisionally term 
'Actuation Dexterity' (AD) that deals with the extent to which numerous specific issues pertaining to the effectiveness and optimization of the actuation and transmission methods, in situations where many DOF are required in a constrained design environment, are collectively addressed. We further propose an approach that can be taken to quantify the actuation dexterity of a device, for quantitative comparison and for design optimization purposes. In this work we use the qualitative description 'Dexterous Actuation' to refer to the application of actuation and transmission methods that exhibit a high degree of actuation dexterity.

\subsection{The Distinction between Dexterity and Actuation Dexterity}

In the engineering literature, dexterity is normally defined at the device level: either as a global property of the device; or as a local device parameter that is a function of the specific configuration of the device (e.g. joint positions); or as a more general parameter that takes into account both the device and the task to be performed. Throughout the last thirty or so years, dexterity as a concept has been applied almost exclusively to describe the performance of devices that are associated with the imitation or extension of the human arm and hand functions. Very often the dexterity of a device is described only in a qualitative manner, however several quantitative definitions of dexterity can be found in the literature.

Thus, the dexterity of a robot arm/wrist system has been defined as its ability to move and apply forces and torques in arbitrary directions as easily and isotropically as possible (e.g. $[10,11])$. A representative point is normally taken on the end effector (e.g. the tool centre point), and the local dexterity, when this point is at a given position within the robot workspace, is expressed in terms of the quantified anisotropy, derived analytically from the manipulator Jacobian. The global dexterity for the system can then be measured by integrating over the workspace [12].

The dexterity of an artificial hand is more difficult to define and quantify because here the issue is not simply one of ease of mobility of a single point on the device, but rather the ability of the device as a whole to impart arbitrary motions and forces on to an arbitrarily shaped object located at an arbitrary position and orientation within the workspace of the hand. The dexterity of a robot hand has been defined qualitatively as the capability of the end effector, operated by a suitable robotic system, to perform autonomously tasks with a certain level of complexity, and the dexterity domain is taken to include grasping and internal manipulation [13]. Various proposals have been made to define a quantifiable measure of the dexterity of an artificial hand, in terms of the range of manipulability of a standard object (e.g. [14]), or in terms of the ease with which a specific task can be performed (e.g. [15]).

Instrumentation for MIS is considered to provide an extension to the function of the human arms and hands, and as such the term dexterity has been applied also to these tools. 
Generally the term has been applied only as a qualitative descriptor for MIS tools, or quantified simply in terms of the number of degrees-of-freedom of motion available at the end effector (e.g. [16]).

The definition of actuation dexterity proposed herein is conceptually different from any of the definitions of dexterity referred to above. Rather than a measure of the overall performance of the device, $A D$ is intended to provide a framework for the evaluation of the detailed actuation approach used in the device. It probes the overall effectiveness and appropriateness of all of the elements of the actuation system, including those pertaining to transmission of actuation forces and torques, for the specific device for which the system is intended. As presented in this work, the concept of AD is intended to apply to devices that are required to carry out motions, and to apply forces/torques, in multiple directions, and that are subject to design constraints of space and weight. Where the device is an artificial hand or an articulated MIS tool, the device itself may also exhibit a high dexterity as defined earlier in this section, and in these cases a high AD will therefore very likely contribute to increased overall dexterity of the device. The concept of AD is however not limited to devices that are associated with the imitation or extension of the human arm and hand functions, but may in principle be applied to any device that has similar constraints. Moreover, since $A D$ is meant to evaluate the actuation system at every level, even properties such as the cost of the actuation elements used (in relation to the average actuator cost for the device type) are taken into consideration in the evaluation.

The concept of $A D$ is intended to provide a dedicated and detailed framework through which the actuation approach applied, or intended to be applied, to a device can be subjected to a supplementary, high level assessment and objective evaluation. This can then lead to the development of design guidelines for the optimization / maximization of $A D$, and therefore for the optimization of the actuation approach with respect to the required performance of the device. It is pointed out that in view of these goals, the AD approach goes far beyond serving simply as an aid to actuator selection, but rather it contributes to a comprehensive evaluation of the entire approach / philosophy taken to the actuation of a device. The intended role, within the device development process, of the protocol for the evaluation and quantification of $A D$, herein referred to as the dexterous actuation assessment protocol or DAAP, is illustrated in Fig. 1. The DAAP is intended to provide input to the conceptual, embodiment and detailed design phases of the development of a MDOF device, and can also contribute to the assessment of existing devices with a view to the development of device upgrades. 


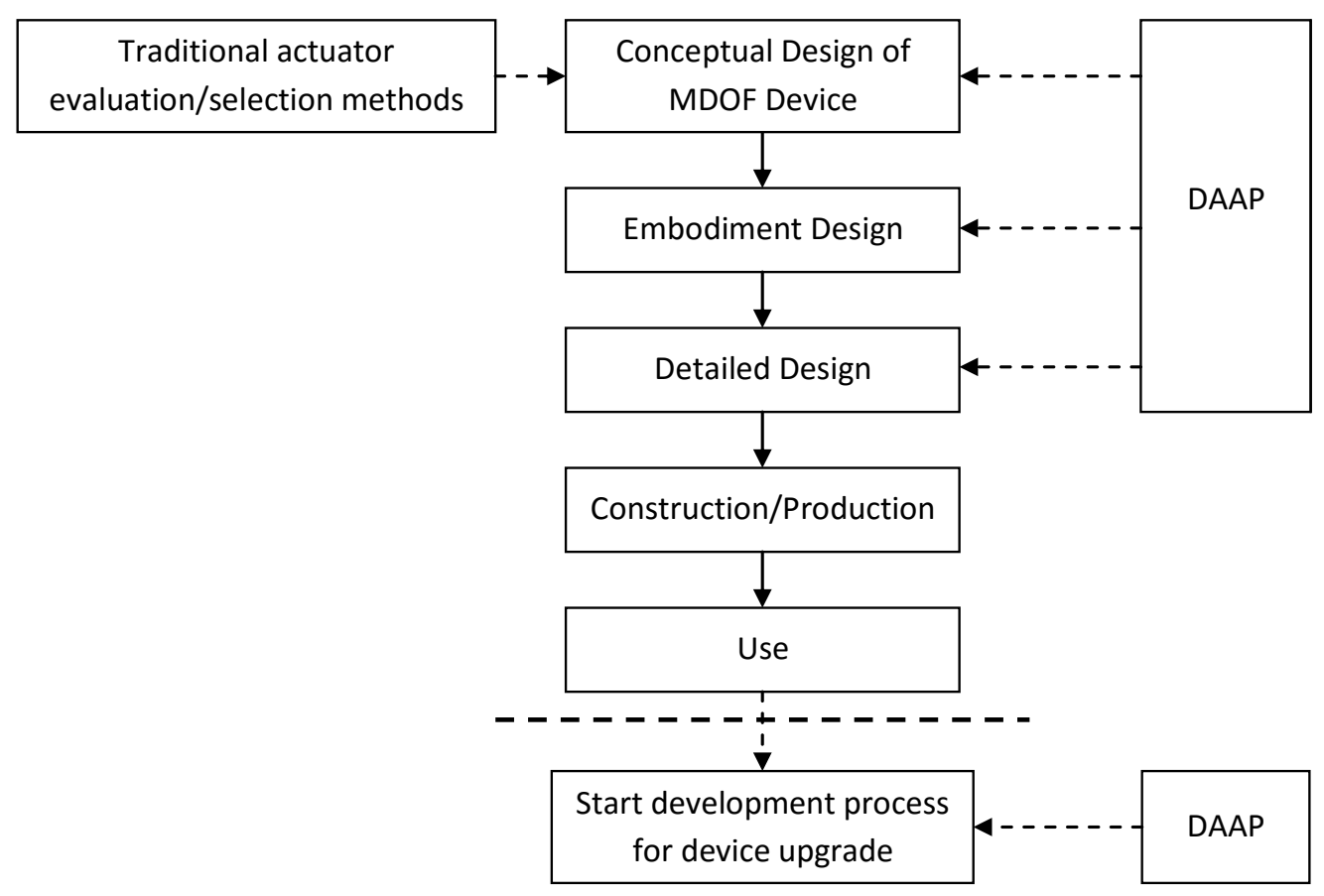

Fig. 1. Expected roles of the DAAP in the development process of a MDOF device

\subsection{Methodology for the Development of a Dexterous Actuation Assessment Protocol}

In this work, the protocol for the evaluation and quantification of $A D$ is developed through an appropriate case study. An example of a device type that is known to require the actuation of multiple DOFs in a constrained environment is an artificial hand, and this device type is therefore considered to be a typical engineering application that would benefit from a study of $A D$. We have therefore opted to use the field of artificial hand development as a vehicle for the development of a generalized DAAP.

The methodology that we have adopted, and that is reported in the remainder of this paper, is as follows. First, we have selected thirty-six different artificial hand projects from among those that are described in considerable detail in the literature, such that the selected projects cover as wide a variety of different actuation methods as possible. We have then carried out an extensive analytical review of these projects, with the aim of compiling and gleaning knowledge on all of the issues pertaining to the actuation problem in artificial hands. Next, we have rationalized this knowledge with respect to the different objectives and expectations of the various projects, in order to seek a level evaluation base with respect to these different designs. This was followed by the identification and development of a number of common evaluation criteria, mainly extracted from issues that were brought up either directly or indirectly by the reviewed projects, and that are relevant to the central problem of dexterous actuation. Some of these criteria are of a quantitative nature, while others are of a qualitative nature. We have taken provisional decisions on the categorization and normalization of these criteria, and on the general scoring method to be used in conjunction with the criteria, with a view to developing a platform that can be used to quantify the $A D$ of any specific artificial hand. This was then followed by the establishment 
of specific scoring details for each individual evaluation criterion. A small subset of the originally reviewed hand projects were then selected to test the approach in detail, and a provisional quantified value of $A D$ was extracted for each of these projects based on the developed protocol and on the available data.

Towards the end of the paper the implications of this study on device design are further elaborated. We also discuss briefly the generality of the evaluation criteria used in the above case study, in the context of their applicability to a different type of device, namely an articulated MIS tool.

\section{Overview of General Actuation Issues in Artificial Hand Projects}

In general, the issues concerning the mechanical design of the actuation system for an artificial hand include those related to (i) the type of actuators and transmission systems; (ii) the number of directly or indirectly driven joints and the organisation of the actuators (actuation architecture); and (iii) the location of the actuators. Actuators may be placed within the end effector itself (directly at the joints, in the finger links, and/or in the palm), and/or in a forearm attached to the hand, or even separately in a more remote location. Concerning actuation architectures, Melchiorri and Kaneko in [1] describe three principal categories of schemes that can be implemented. For a hand with $m$ actuators and $n$ joints, the three main categories involve (i) the $m<n$ approach where some joints are passive, or kinematically coupled in a fixed or variable way to a controlled joint; (ii) the $m=n$ approach where each joint has its own actuator; and (iii) the $m>n$ approach where more than one actuator acts on a joint.

As regards the types of actuators, amongst the actuator technologies encountered in artificial hand literature, there are the conventional types of actuators, including electromagnetic motors, hydraulic, and pneumatic actuators, and the non-conventional types of actuators. These latter include shape memory alloy (SMA) actuators, ultrasonic motors (USM), pneumatic artificial muscles (PAM), and flexible fluidic actuators (FFA).

Concerning the design of hands, Carrozza et al. [17] and Kawabuchi [18] describe two research directions. One line of research seeks to attain motion and functional capabilities that are similar to those of the human hand, with the implementation of many powerful actuators, and complex control schemes. This approach is typically adopted in hands intended for research in grasping and manipulation. This type of hand tends to be larger than the human one, sometimes even when the actuators are located away from the hand [18].

The second line of research emphasizes similarity in size and weight to the human hand by reducing the mass, size, and/or number of actuators. This type of approach is applied in devices such as prosthetic hands where in addition to appearance, significant importance is given to low energy consumption and simplicity of control [17]. To mitigate the limited 
functionality resulting from this approach, researchers adopt specific solutions. For example to improve the dexterity of traditional prosthetic hands and simultaneously respect the mass and size requirements, Carrozza et al. [19] proposed a hand with more, but less powerful actuators.

\section{The Reviewed Hands and a Breakdown of their Main Features}

The thirty-six reviewed hand projects are listed in Table 1. The declared applications of these artificial hands are given in Fig. 2 (some projects indicated multiple applications). Fig. 3 gives breakdowns of the hands according to number of digits; type of mechanical framework; structural integration; actuation architecture; actuator technology; and transmission elements used. Fig. 4 gives a breakdown of the mechanisms used where passive joints were coupled to active joints in the hands.

A significant problem that we have faced in this work pertains to the unavailability of many elements of key data on many of the reviewed projects, and also to the lack of standardization of data even where these are reported. We have described this problem in detail in [54]. Often, we have been able to fill in many of the missing gaps to varying extents by consulting various publications on each project; by extrapolating the provided information; by comparing to similar situations in other devices/fields; by referring to the equipment (e.g. actuator) manufacturer datasheets where these were available; and/or by corresponding with the project researchers.

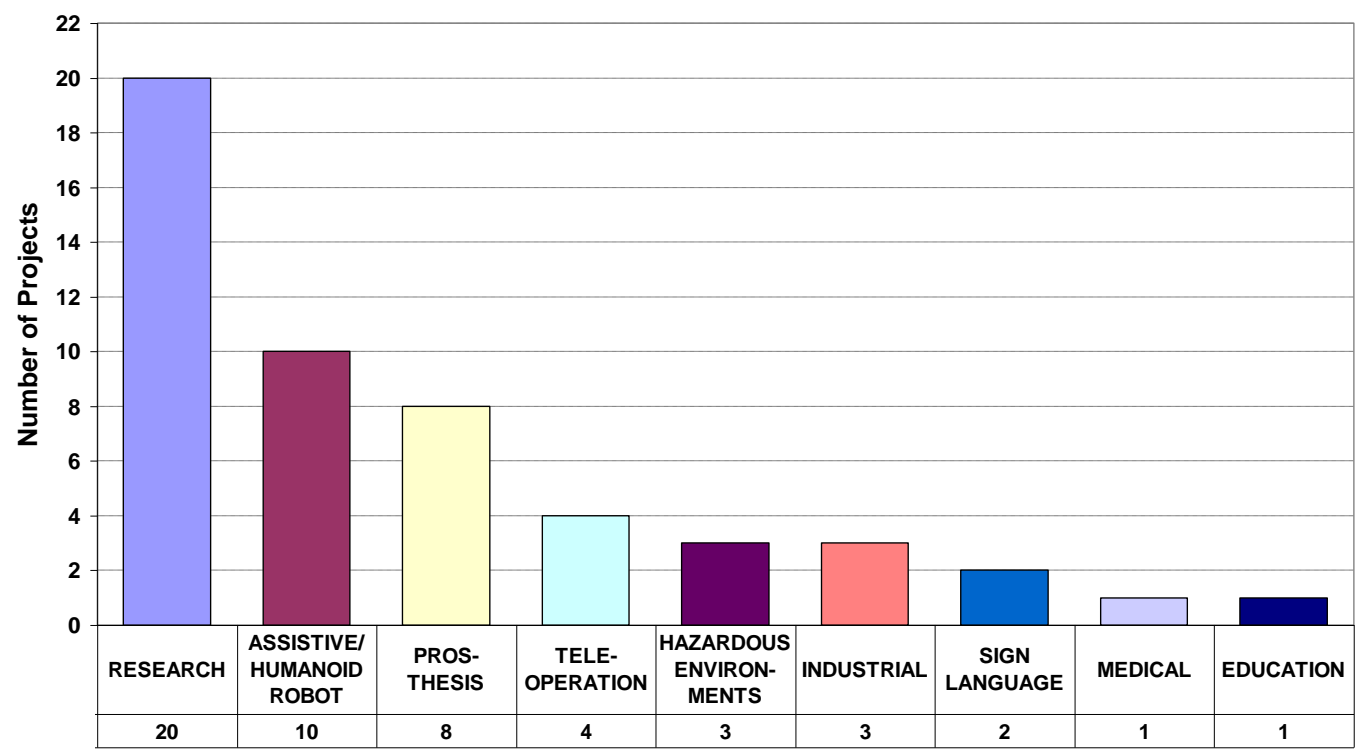

Fig. 2. Fields of application of the reviewed projects 


\begin{tabular}{|c|c|c|c|c|}
\hline Ref & Device Name (or paper title) & Year & PP* & Organisation \\
\hline 1 & The BarrettHand Grasper - BH8-250 & 2000 & {$[20]$} & Barrett Technology Inc., U.S.A. \\
\hline 2 & The MANUS Hand & 2004 & {$[8]$} & $\begin{array}{l}\text { Consortium: European Commission TELEMATICS } \\
\text { Programme } \\
\end{array}$ \\
\hline 3 & The DLR Hand I & 1998 & {$[21]$} & \multirow{2}{*}{ German Aerospace Research Center (DLR), Germany } \\
\hline 4 & The DLR Hand II & 2001 & {$[22]$} & \\
\hline 5 & The DLR/HIT Hand I & 2008 & {$[23]$} & \multirow{2}{*}{$\begin{array}{c}\text { German Aerospace Research Center (DLR), and } \\
\text { Harbin Institute of Technology, China (HIT) }\end{array}$} \\
\hline 6 & The DLR/HIT Hand II & 2008 & {$[24]$} & \\
\hline 7 & The GIFU Hand II & 2002 & {$[25]$} & \multirow{4}{*}{ GIFU University, Japan } \\
\hline 8 & The GIFU Hand III & 2002 & {$[26]$} & \\
\hline 9 & Kinetic Humanoid $(\mathrm{KH})$ Hand & 2004 & {$[27]$} & \\
\hline 10 & Kinetic Humanoid Hand Type S & 2005 & {$[28]$} & \\
\hline 11 & Robot Hand using Ultrasonic Motors & 2005 & {$[29]$} & \multirow{2}{*}{ Keio University, Japan } \\
\hline 12 & Miniature SMA Actuated Hand & 2006 & {$[30]$} & \\
\hline 13 & Robotic Hand with Vast DOF Artificial Muscle Actuators & 2006 & {$[31]$} & Massachusetts Institute of Technology (M.I.T.), U.S.A. \\
\hline 14 & The NAIST-Hand - eNAIST-Handf & 2005 & {$[32]$} & Nara Institute of Science and Technology (NAIST), Japan \\
\hline 15 & The NASA Robonaut Hand & 1999 & {$[33]$} & NASA, U.S.A. \\
\hline 16 & Hand for HRP-3P Humanoid Robot & 2007 & {$[34]$} & $\begin{array}{c}\text { National Institute of Advanced Industrial Science and } \\
\text { Technology (AIST), Japan }\end{array}$ \\
\hline 17 & Pneumatic Soft Robot Hand for Human Friendly Robot & 2003 & {$[35]$} & Okayama University, Japan \\
\hline 18 & The Ultralight Anthropomorphic Hand & 2001 & {$[36]$} & \multirow{3}{*}{ Research Center of Karlsruhe (FZK), Germany } \\
\hline 19 & Anthropomorphic Hand for a Mobile Assistive Robot & 2005 & [37] & \\
\hline 20 & Multifunctional Cosmetic Hand Prosthesis - Fluidhand P4 & 2006 & {$[38]$} & \\
\hline 21 & The SPRING Hand & 2004 & {$[17]$} & \multirow{2}{*}{ Scuola Superiore Sant'Anna, Italy } \\
\hline 22 & The CyberHand & 2006 & {$[39]$} & \\
\hline 23 & The Shadow Dexterous Hand C5 & 2008 & {$[40]$} & \multirow{2}{*}{ Shadow Robot Company, U.K. } \\
\hline 24 & The Shadow Dexterous Hand C6M & 2009 & {$[41]$} & \\
\hline 25 & The UB Hand III & 2005 & {$[42]$} & University of Bologna, Italy \\
\hline 26 & The LARM Hand & 2004 & {$[43]$} & University of Cassino, Italy \\
\hline 27 & The DIST Hand & 2000 & {$[44]$} & University of Genoa, Italy \\
\hline 28 & The IAL Hand & 2007 & {$[45]$} & University of Malta, Malta \\
\hline 29 & The Southampton-Remedi Hand & 2000 & {$[46]$} & University of Southampton, U.K. \\
\hline 30 & Artificial Hand Actuated by Pneumatic Artificial Muscles & 1999 & {$[47]$} & \multirow{3}{*}{ University of Tokyo, Japan } \\
\hline 31 & Multi-DOF Electromyography Prosthetic System & 2006 & {$[48]$} & \\
\hline 32 & $\begin{array}{l}\text { Anthropomorphic Robot Hand with Backdrivable Hydrostatic } \\
\text { Transmission }\end{array}$ & 2007 & {$[49]$} & \\
\hline 33 & Child Sized Prosthetic Hand & 1999 & {$[50]$} & \multirow{2}{*}{ University of Toronto, Canada } \\
\hline 34 & Shape Memory Alloy Based Dexterous Robot Hand & 2007 & {$[51]$} & \\
\hline 35 & The Pinching Hand & 2005 & {$[52]$} & University of Tsukuba, Japan \\
\hline 36 & The Utah/MIT Dextrous Hand (III) & 1986 & {$[53]$} & University of Utah / M.I.T., U.S.A. \\
\hline
\end{tabular}

* principal publication

Table 1. The reviewed artificial hand projects

\section{The Approach to Dexterous Actuation}

\subsection{The Conceptual Challenge}

Even if we consider just the general device type of 'artificial hands', the review of the literature, as seen in section 4 above, unveils a wide variety of different devices with different properties, intended for different specific objectives, and developed through different design strategies and approaches. When we extend our consideration to include also other device types that exhibit MDOF under space and weight constraints, this variety increases further. 


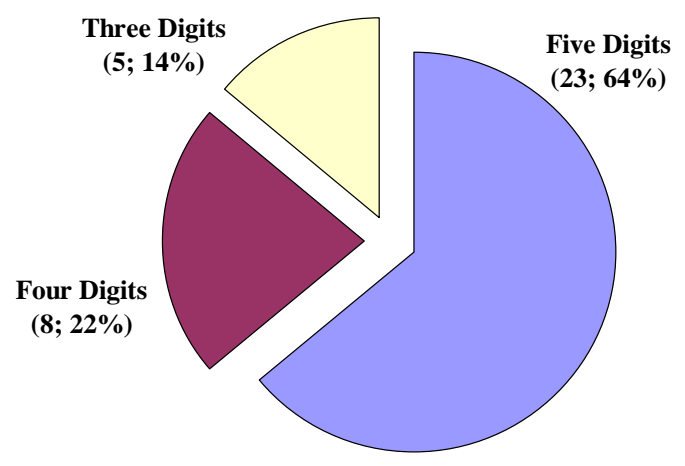

(a) Number of digits

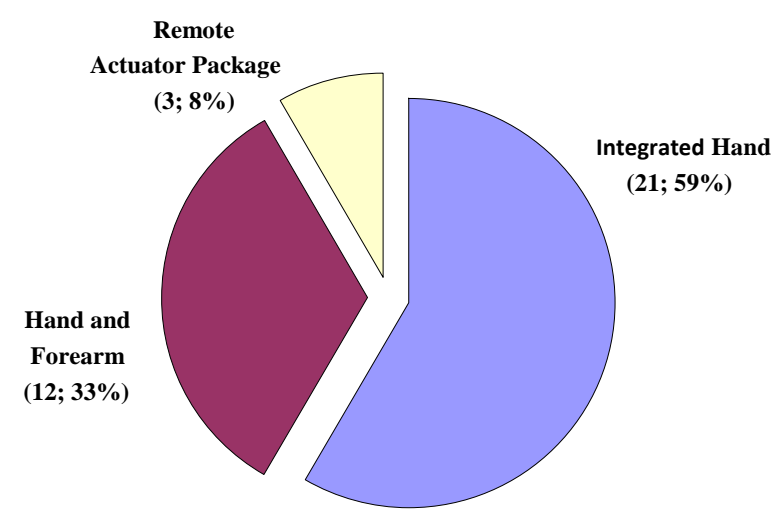

(c) Type of structural integration

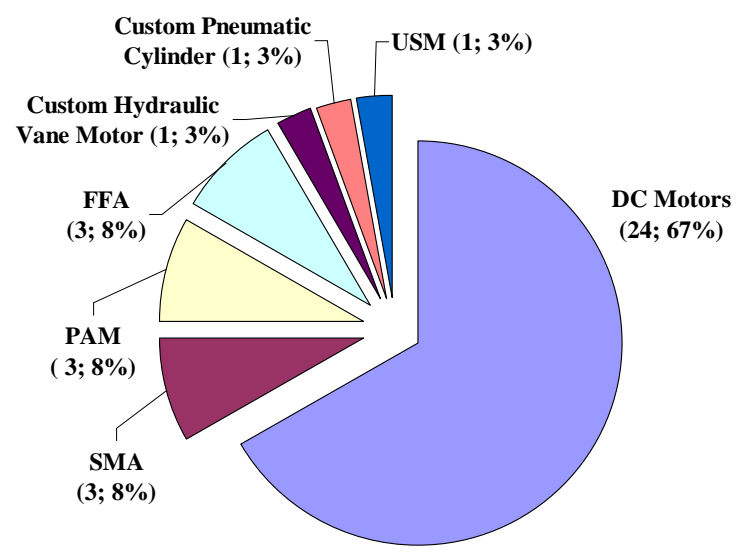

(e) Actuator technologies

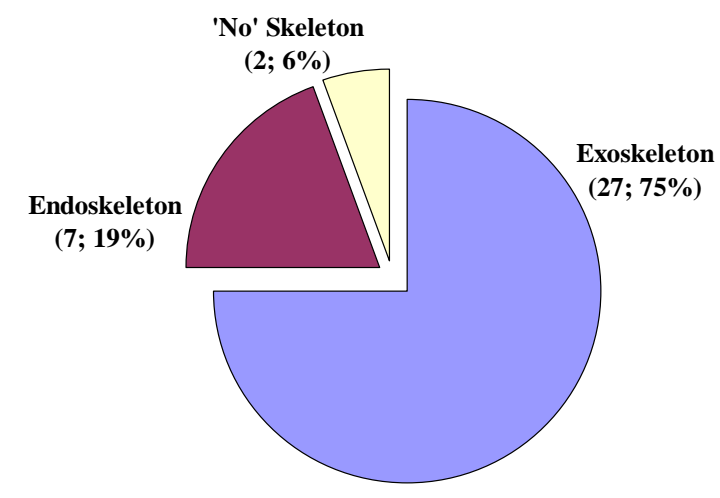

(b) Types of mechanical framework

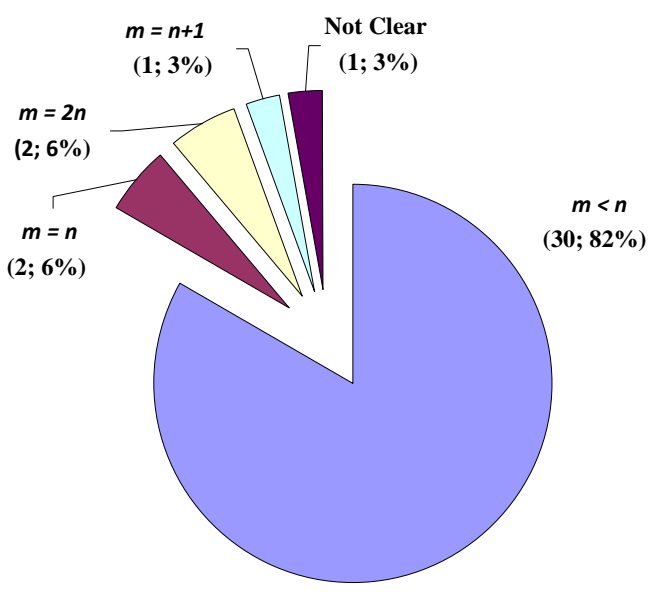

(d) Actuation architectures

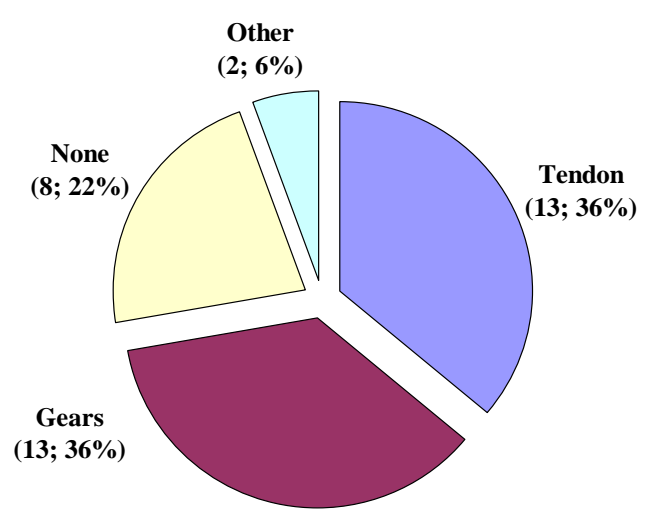

(f) Main transmission elements

Fig. 3. Breakdown of the main features of the reviewed projects

The objective that was set in this work is to define one general measure that can be used to assess, in an overall and strongly indicative way, the effectiveness of the actuation and transmission system of any such device not only with respect to the intrinsic capabilities of this system, but also with respect to the higher level properties and objectives of the device.

Thus it was important that the evaluation criteria to be used should be both universal in nature, such that they can be applied to all of the devices and device types under 
consideration; and normalized, in order for the scoring methods used for each criterion to be widely applicable without undue need for adjustment when investigating different devices or device types.

Coupling Mechanisms and Methods

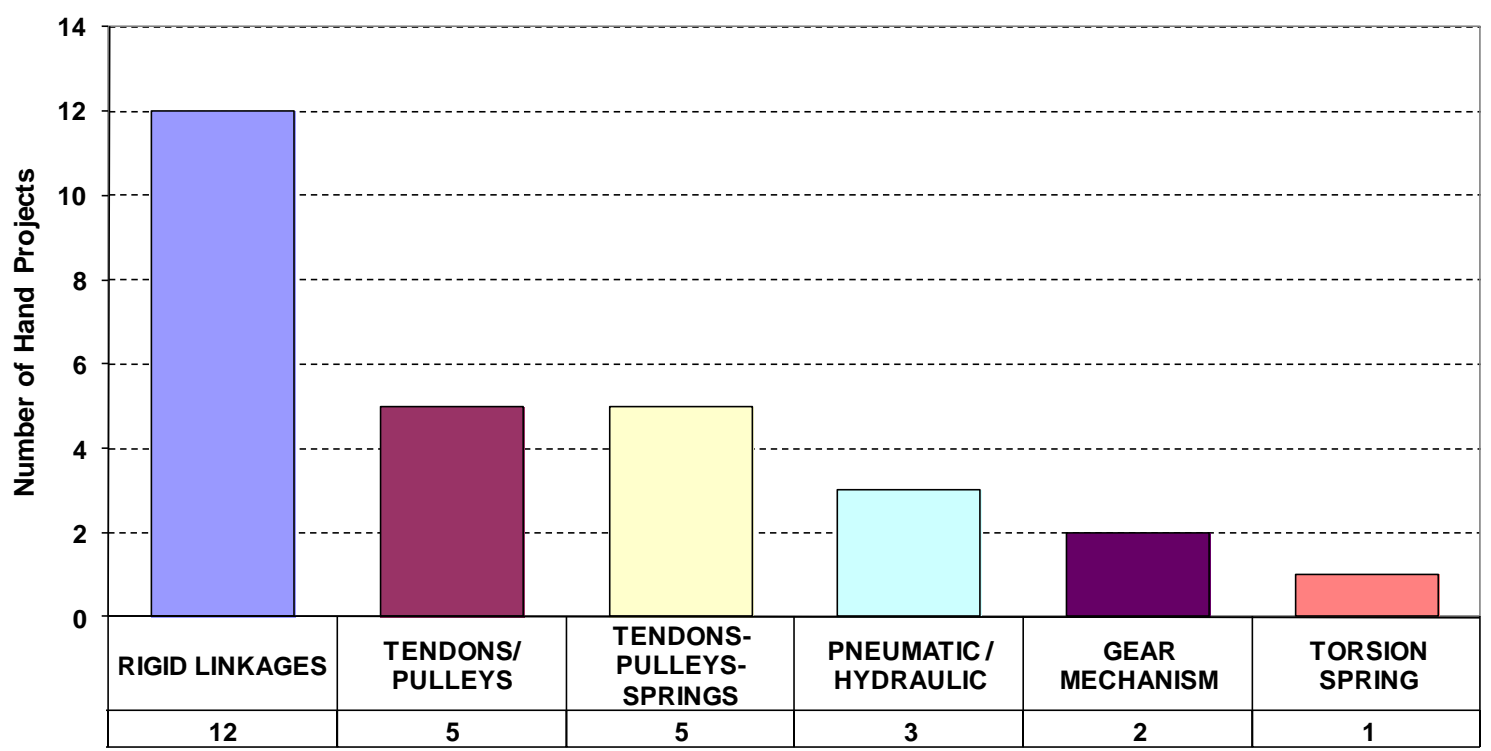

Fig. 4. Coupling mechanisms for the passive joints

\subsection{Universality and Normalization Measures}

Based on the reviewed literature, we have condensed the features of devices that exhibit MDOF in a constrained environment to a number of generalized universal elements, and we have abstracted these as shown in the left hand column of Fig. 5. Under this representation, and for a specific device type, the relevant constituent elements, properties and / or manifestations of each of these universal elements can be derived from a defined subset of possibilities. The right hand column of Fig. 5 shows specific subsets that apply to artificial hands.

One of these universal elements, as defined in this work, is the actuation system. This can be considered to consist of the actuator(s) themselves, of the transmission system(s) (if any exist), and of the dedicated support structure(s) (if any exist). The set of general overall properties of the actuation system is also here considered to be a separate constituent element of the actuation system. We can go one step down the hierarchy of this representation, and depict these constituent elements as the universal elements of the actuation system, as shown in the left hand column of Fig. 6. The relevant constituent elements, properties and / or manifestations of each of these new universal elements are shown in the right hand column of Fig. 6. 


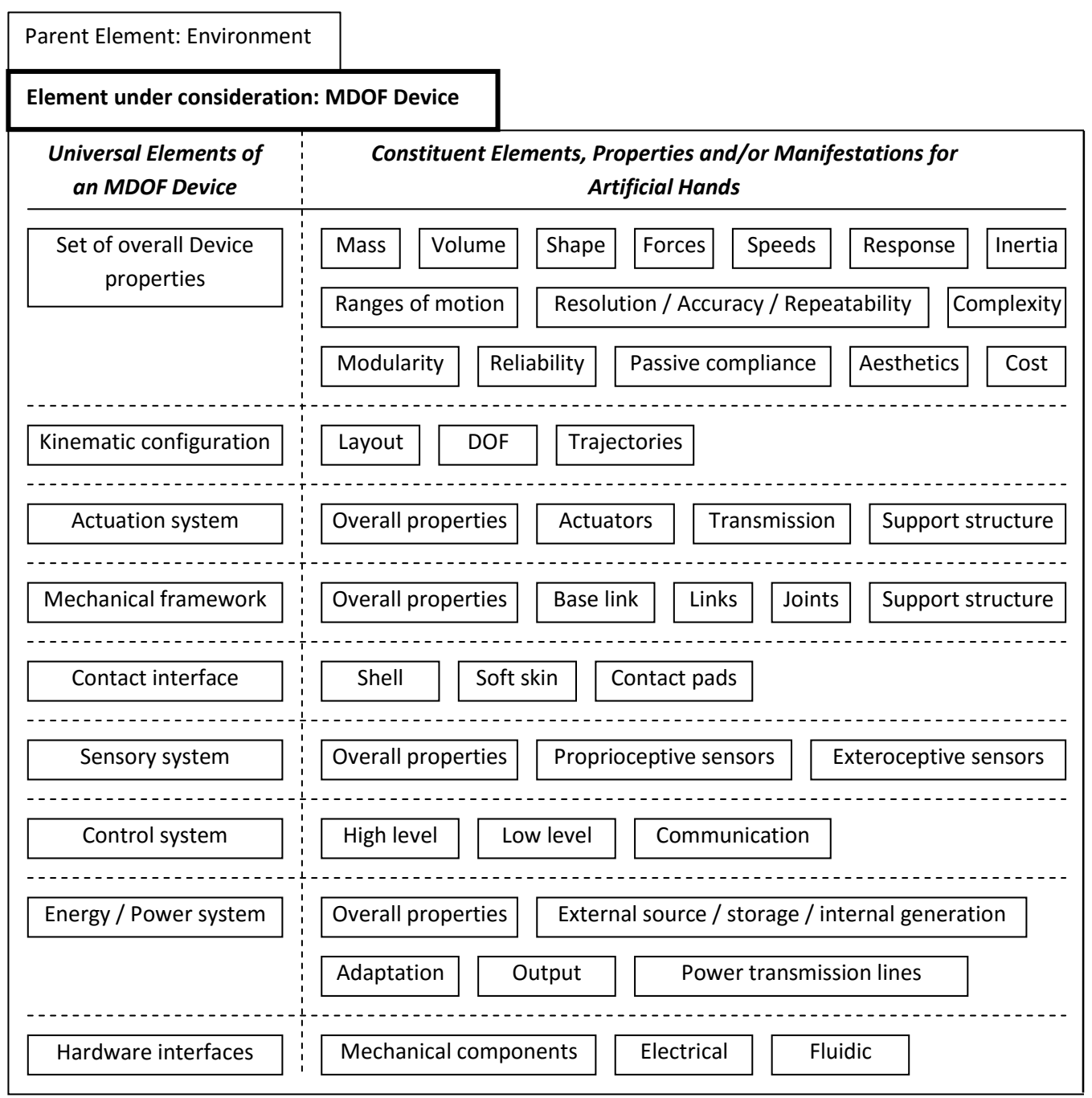

\section{Fig. 5. Universal elements of a MDOF device}

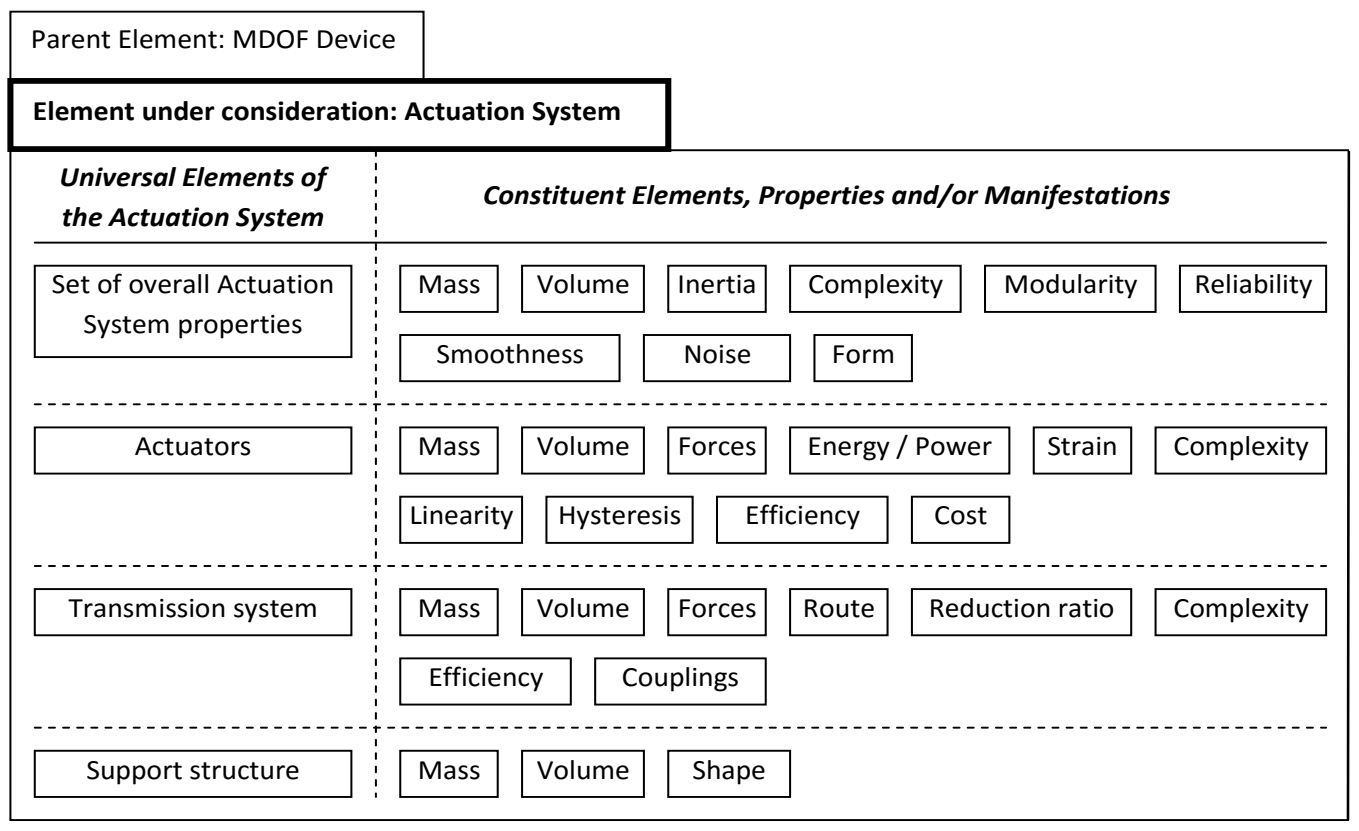

Fig. 6. Universal elements of the actuation system 
We have developed the set of $A D$ evaluation criteria by examining the universal and constituent elements of the actuation system; their interaction with the other universal elements of the device; as well as the extent to which they satisfy the expectations of the device type. Before acceptance, each criterion was checked for applicability to the central problem, and in general this acceptance was based on the issues that were extracted from the reviewed projects. It is noted that where the physical properties of an actuator are concerned, we have considered the entire implemented unit. Thus for example, as reported in [55], although the power to mass ratio of modified NiTi SMA fibres could reach nearly 50 $\mathrm{kW} / \mathrm{kg}$, when implemented as a SMA actuator for a robot hand the integrated actuator was found to produce only $6 \mathrm{~W} / \mathrm{kg}$. Peirs et al. in [56] reported similar findings concerning the construction of SMA driven devices for endoscopy.

Normalization of the criteria was addressed in two ways. Firstly, all of the quantitative criteria have been non-dimensionalized. Where it was not possible to extract a nondimensionalized parameter with respect to an inherent attribute of the device, we have non-dimensionalized the criterion with respect to the equivalent parameter for the human hand, taken therefore as the reference standard for these criteria. We feel that the use of the human hand as a reference is justified since this organ remains the quintessential light and compact MDOF system, and it is therefore significant to relate and contrast the properties of artificial MDOF systems (even where the device type is not an artificial hand) to those of this natural system. We have avoided the non-dimensionalization of forces with respect to weights, in order to maintain direct applicability of the AD measure also to devices that operate outside the Earth.

The second way in which we have introduced normalization, is that where applicable, the qualitative criteria have been defined in terms of the properties or objectives of the device under consideration. Thus for example, when investigating the noise level of an actuation system, this would be evaluated in relation to the noise requirements / expectations of the overall device. In this regard it is emphasized that the aim of this study is not to investigate the appropriateness of a particular general device design (e.g. the decision to have three fingers, with three links per finger made of aluminium, in an artificial hand) in relation to the required task, but rather to investigate the appropriateness of the actuation approach given the general device design and the overall device properties and expectations. As such, many of the investigated properties of the actuation system must for comparison purposes be normalized to the specific design and properties of the device.

It is noted that since the overall $A D$ measure evaluates the total actuation solution with respect to its application to the specific device under consideration, then the nondimensional representation of the individual criteria does not necessarily lead to global scale-independent results for a specific type of device. Thus, for example, if a device is scaled down in size while retaining the same type of actuation technology (e.g. electric motors), its score in criteria such as actuator specific power or actuator efficiency may 
change due to an inherent size dependence of these attributes for the specific actuation technology under consideration. Such changes in score may indicate that, for a dimensionally scaled-down version of the same device, a different approach to the actuation system (including choice of actuation technology) may be warranted.

\subsection{Categorization of AD Evaluation Criteria}

In view of the above considerations, we have categorized the evaluation criteria for $A D$ as follows:

1. Intrinsic properties of the actuation system and its constituents

2. Properties / effects of the actuation system with respect to the other universal elements of the device (excluding overall device properties)

3. Properties / effects of the actuation system with respect to the properties and capabilities of the device

4. Properties / effects of the actuation system with respect to the expectations of the device type

\subsection{The Quantification of Actuation Dexterity}

This work has led to the identification of a large set of forty-eight non-dimensional numerical and qualitative evaluation criteria related to the overall appropriateness and effectiveness, at various levels of subtlety, of the actuation approach in situations where many DOF are required in a constrained environment. The criteria are assigned equal weight, although it is noted that certain influential attributes (such as the presence of a transmission, or actuation system mass) of the system appear in multiple criteria and therefore give a greater contribution to the $A D$ score. In quantifying the $A D$ of a given device, we assign a score of either 2, 1, or 0 for each criterion based on the general qualitative definitions given in Table 2. These definitions are amplified and clarified for each specific criterion, based on objective reasoning and on the reviewed literature. Where the criterion is numerical in nature, specific numerical boundaries to separate the three scores are put forward.

\begin{tabular}{|c|l|}
\hline Score & \multicolumn{1}{|c|}{ Description } \\
\hline 2 & Good or Well Addressed or Not Applicable \\
\hline 1 & Fair or Somewhat Addressed \\
\hline 0 & Poor or Not / Minimally Addressed \\
\hline
\end{tabular}

Table 2. General evaluation criteria scores

The question of differences in importance / criticality between the various criteria is one that warrants some discussion. In particular, if different weights are assigned to the criteria these weights may tend to be subjective, and they may also depend on the particular device type or on the specific application, therefore detracting from the universality of the measure. Thus, in line with the intended nature of the proposed measure which, as 
presented here, is intended to function mainly as a driver for optimization and for innovative thinking rather than as a stand-alone actuator selection tool or as a stand-alone measure of actuation system suitability, we have proposed to give all criteria an equal weight. This maximizes the objectivity of the AD measuring system. Where one criterion is more critical to device success than another, this will be reflected in the traditional design evaluation exercises that are carried out in parallel with the DAAP exercise, and in particular any clearly unsuitable designs will be rejected outright through the former exercises. In the cases where a design has not been rejected outright in this manner, the scores assigned to such critical criteria in the DAAP exercise will then reflect the extent to which the issues associated with each of these criteria have been pushed to their optimum.

It is noted that in an alternate application of this approach, customized weighting factors can be introduced for the criteria as part of the product design specification of a specific device. In such an application, the modified DAAP could indeed serve as the primary tool for actuation system selection for the specific device under consideration. In this case, a fourth score alternative ("F" for FAIL) for each criterion would be required in addition to the three scores listed in Table 2, indicating that a design should be rejected irrespective of the scores obtained in the other criteria. In such an application, rather than being assigned universal $A D$ scores, the candidate designs would be evaluated according to their customized DAAP scores.

The application of a basic scale that assigns only three possible scores (Table 2) for each criterion is a recognition of the fact that the criteria are either intrinsically qualitative, or are defined to be so, or are quantifications of attributes whose influence on AD cannot be measured precisely. As such, an attempt to implement a resolution to the individual criteria beyond good/fair/poor may tend to be artificial and/or subjective. This approach also serves to maximize the portability of the AD measuring system between devices and device types. The resolution of the overall AD measure is derived from the large number of criteria that contribute to the total score. An underlying premise of this method is that if one total actuation approach has features that make it potentially better than an alternative approach, this is likely to be faithfully reflected when the two solutions are interrogated and compared under a sufficiently large number of relevant criteria.

\section{The AD Evaluation Criteria}

\subsection{Overview}

Each one of the 48 evaluation criteria for AD is described very briefly below. The setting of the three score partitions for each criterion has been guided by one or more of the following: existing technological possibilities; achievements reported in the reviewed literature; the interpretation of the reviewed literature; the even spreading of the reviewed hands across the three scores; and the characteristics of the human hand. Generally, the overall $A D$ score is anchored to current technological capability, and for any device, the 
measured $A D$ reflects the effectiveness of the actuation system with respect to what is currently potentially achievable.

\subsection{Intrinsic properties of the actuation system}

C1.1 - Specific power of the actuator. This parameter is non-dimensionalized with respect to the specific power of the human muscle, taken as $50 \mathrm{~W} / \mathrm{kg}$ [57]. Score 0: C1.1 $\leq .5$; Score 1: $0.5<\mathrm{C} 1.1 \leq 5$; Score 2: C1.1 > 5 .

C1.2 - Volumetric power of the actuator. This parameter is non-dimensionalized with respect to the typical volumetric power of the human muscle, estimated (and rounded) from [57] to be $0.05 \mathrm{~W} / \mathrm{cm}^{3}$. Score 0: $\mathrm{C} 1.2 \leq 20$; Score 1: $20<\mathrm{C} 1.2 \leq 2000$; Score 2: C1.2 > 2000.

C1.3 - Maximum actuator strain. Score 0: C1.3 $\leq .1$; Score 1: $0.1<\mathrm{C} 1.3 \leq 0.5 ;$ Score 2: C1.3 $>0.5$. For infinite rotary actuators (e.g. rotary DC motor), the maximum score is applied.

C1.4 - Force consistency / linearity along stroke. Score 0: significantly variable and nonlinear; Score 1: linear or close to constant; Score 2: constant.

C1.5 - Actuator hysteresis. Score 0: considerable; Score 1: minor; Score 2: none.

C1.6 - Actuator efficiency. Score 0: C1.6 $\leq$ 0.33; Score 1: $0.33<\mathrm{C} 1.6 \leq 0.67$; Score 2: C1.6 > 0.67 .

C1.7 - Actuator complexity. Complexity is evaluated qualitatively. The final state of packaging of the actuator is considered in view of the facility of implementation. Concerning the construction, this criterion focuses principally on custom designed and built actuators, while considers commercially available actuators as using well-proven solutions. Score 0: reported or considered to be relatively complex; Score 1: reported or considered to be of average complexity; Score 2: reported or considered to be relatively simple.

C1.8 - Actuator implementation in conjunction with a mechanical transmission. Score 0: implemented with a transmission; Score 1: implemented without a transmission, but requires a distinct joint; Score 2: implemented without a transmission and utilized directly as a joint.

C1.9 - Complexity of the transmission train. The evaluation takes into account both the number of stages and the number of different element sets used in the transmission. For the purpose of this criterion, we have defined complexity as the product of these two numbers, based on the most complex transmission train in the device. Where there is no transmission system, the maximum score is applied. Score 0: C1.9 $\geq 7$; Score 1: $6 \geq$ C1.9 $\geq 4$; Score 2: $\mathrm{C} 1.9 \leq 3$.

C1.10 - Transmission route across device components. The score is based on the longest transmission train in the device, and where there is no transmission system, the maximum 
score is applied. Score 0: transmission starts from a location remote to the end-part or forearm; Score 1: transmission spans across several links, but remains within the end-part or forearm; Score 2: transmission is within an adjacent link, or not applicable.

C1.11 - Interference between transmission systems of different actuators. Score 0: significant interference reported or apparent; Score 1: some degree of interference reported or apparent; Score 2: no or negligible interference reported or apparent, or not applicable.

C1.12 - Inertia of the moving components of the transmission system. Score 0: designed to have relatively significant concentrated masses located distally; Score 1: designed to have distally concentrated masses that are relatively light in weight; Score 2: designed to minimize inertia, or no significant concern reported or apparent.

C1.13 - Mass of the actuators in relation to the mass of the actuation system. This criterion, and the next one, reward systems that consist of a larger proportion of active actuator material as opposed to transmissions and supporting structures. Score 0: C1.13 $\leq$ 0.33; Score 1: $0.33<\mathrm{C} 1.13 \leq 0.67$; Score 2: C1.13 > 0.67 .

C1.14 - Volume of the actuators in relation to the volume of the actuation system. Score 0: C1.14 $\leq$ 0.33; Score 1: $0.33<\mathrm{C} 1.14 \leq 0.67$; Score 2: C1.14 > 0.67 .

C1.15 - Internal resistance to motion. The evaluation takes into consideration any reported or perceived resistance to motion caused by friction and the compression/extension of elastic elements such as springs in the transmission. Score 0 : significant resistance reported or apparent; Score 1: some resistance reported or apparent; Score 2: significant efforts to minimise resistance reported, or low resistance reported or apparent.

C1.16 - Intrinsic energy saving features provided by the actuation system. This criterion evaluates the ability of the device links to maintain position or force without the supply of energy, due to the characteristic properties of either or both of the actuator and the transmission system. Score 0 : reported or expected to need energy to maintain the link positions; Score 1: reported to maintain the link positions without the supply of energy; Score 2: reported to maintain also the link forces without the supply of energy, or not applicable.

C1.17 - Similarity among the actuators. When viewed in isolation of other factors, similarity among the actuators is considered beneficial to the actuation system design in terms of general simplicity. Score 0: different types; Score 1: same types, but different models; Score 2: identical actuators.

C1.18 - Modularity, and ease of maintenance / replacement of the actuation system. Score 0 : all or many actuation trains are not modular and/or difficult to maintain; Score 1: a few 
actuation trains are not modular and/or somewhat difficult to maintain; Score 2: all actuation trains are modular and/or can be maintained with ease.

C1.19 - Reliability / robustness of the actuation system. Score 0: severe limitations disclosed or known to exist; Score 1: some limitations disclosed or known to exist; Score 2: given considerable attention or known to be relatively reliable.

C1.20 - Smoothness of actuation system motion. This criterion evaluates 'stick-slip' effects, excessive vibration and noise, and instantaneous mechanical jerks during motion. Score 0 : reported or expected not to be smooth; Score 1: reported or expected to be only somewhat smooth; Score 2: reported or expected to be smooth.

\subsection{Properties / effects of the actuation system with respect to the other universal elements of the device}

C2.1 - Commonality of energy supply. Score 0: the actuation system uses more than one type of supply, or a type of supply that is different from that of all the other device elements; Score 1: as above, but utilizes an in-built conversion unit that uses the same type of supply as other device elements; Score 2: the actuation system uses one type of supply, which where applicable is the same as that used by at least one other device element.

C2.2 - Actuator requirement for internal sensors. This criterion is included in order to recognize efforts to reduce the number of sensors needed by the actuation system. Score 0 : the actuation system is not able to fulfil the basic functional requirements of the device without internal feedback sensors; Score 1: there is limited capability to fulfil the basic functional requirements without internal sensors (e.g. only position control); Score 2: the actuation system is able to fulfil the basic functional requirements without internal sensors.

C2.3 - Adaptability of kinematically coupled joints. This criterion evaluates whether the motion relationships between coupled joints in the device can be adjusted. Score 0: all applicable joints are coupled in a fixed way; Score 1: some of the applicable joints are coupled with adaptable mechanisms; Score 2: all applicable joints are coupled with adaptable mechanisms, or not applicable.

C2.4 - Mass of the actuation system in relation to the number of controlled DOFs. The mass of the actuation system is normalized with respect to the end-part mass (criterion C3.1), and then divided by the number of DOFs. Score 0: C2.4 >0.2; Score 1: $0.1<$ C2.4 $\leq 0.2$; Score 2: C2.4 $\leq 0.1$.

C2.5 - Volume of the actuation system in relation to the number of controlled DOFs. The volume of the actuation system is normalized with respect to the end-part volume (criterion C3.2), and then divided by the number of DOFs. Score 0: C2.5 >0.2; Score 1: $0.1<\mathrm{C} 2.5 \leq 0.2$; Score 2: $\mathrm{C} 2.5 \leq 0.1$. 
C2.6 - Mass of the actuation system in relation to the total number of joints. The mass of the actuation system is normalized with respect to the end-part mass (criterion C3.1), and then divided by the number of joints. Score 0: C2.6 > 0.1; Score 1: $0.05<\mathrm{C} 2.6 \leq 0.1$; Score 2: C2.6 $\leq 0.05$.

C2.7 - Volume of the actuation system in relation to the total number of joints. The volume of the actuation system is normalized with respect to the end-part volume (criterion C3.2), and then divided by the number of joints. Score 0: C2.7 > 0.1; Score 1: $0.05<$ C2.7 $\leq 0.1$; Score 2: $\mathrm{C} 2.7 \leq 0.05$.

\subsection{Properties / effects of the actuation system with respect to the properties and capabilities of the device}

C3.1 - Mass of the actuation system in relation to the mass of the end-part of the device. Where the actuators are an integral part of the device they are included as part of the device mass, otherwise if they are located outside the end-part they are not. Score 0: C3.1 > 2; Score 1: $0.5<$ C3.1 $\leq$ 2; Score 2: C3.1 $\leq 0.5$.

C3.2 - Volume of the actuation system in relation to the volume of the end-part of the device. Where the actuators are an integral part of the device they are included as part of the device volume, otherwise if they are located outside the end-part they are not. Score 0 : C3.2 > 2; Score 1: $0.5<$ C3.2 $\leq 2$; Score 2: C3.2 $\leq 0.5$.

C3.3 - Maximum end-part exerted force in relation to the mass of the actuation system. This parameter is non-dimensionalized with respect to the ratio for the human hand system, estimated at $100 \mathrm{~N} / \mathrm{kg}$ based on [58] and [59]. Score 0: C3.3 $\leq .5$; Score 1: $0.5<$ C3.3 $\leq 2.5$; Score 2: C3.3 > 2.5.

C3.4 - Maximum end-part exerted force in relation to the volume of the actuation system. This parameter is non-dimensionalized with respect to the ratio for the human hand system, estimated at $0.1 \mathrm{~N} / \mathrm{cm}^{3}$ based on [58] and [59]. Score 0: C3.4 $\leq 1.0$; Score 1: $1.0<$ C3.4 $\leq$ 10.0; Score 2: C3.4 > 10.0.

C3.5 - Maximum end-part exerted force in relation to the maximum internal force. Score 0: C3.5 $\leq$ 0.1; Score 1: $0.1<$ C3.5 $\leq$ 1; Score 2: criterion not applicable or not significant.

C3.6 - Contribution of the actuation system to end-part resolution, accuracy, and repeatability. The evaluation takes into consideration the properties and possibilities provided by the actuators (e.g. the resolution attributed to the type of actuator), the transmission (e.g. the reduction ratio), and the related sensors (e.g. the location of the position sensors), to achieve these performance attributes. Score 0: limited; Score 1: reasonable; Score 2: good.

C3.7 - Effects of the actuation system on the aesthetics of the device. In the case of artificial hands, this is taken to refer to the degree to which the actuation system causes the hand to 
deviate from the shape, size and general aspect of the human hand. Score 0: significant effects reported or apparent; Score 1: some effects reported or apparent; Score 2: no or negligible effects apparent, or not applicable.

\subsection{Properties / effects of the actuation system with respect to the expectations of the device}

C4.1 - Achievable device joint speeds. This parameter is non-dimensionalized with respect to the maximum speed of the human hand joints, rounded to $1000 \% \mathrm{~s}$ based on [60] and [61]. Score 0: C4.1 $\leq .1$; Score 1: $0.1<$ C4.1 $\leq .4$; Score 2: C4.1 >0.4.

C4.2 - System response (time constant or equivalent) of the device. This parameter is nondimensionalized with respect to the time constant for human hand joint motion, estimated and rounded to $100 \mathrm{~ms}$ (neural and muscle delays) based on [62] and [63]. Score 0: C4.2 2 5; Score 1: $1<\mathrm{C} 4.2<5$; Score 2: $\mathrm{C} 4.2 \leq 1$.

C4.3 - Position resolution/controllability of the device as afforded by the actuation system. Score 0: low; Score 1: reasonable; Score 2: high.

C4.4 - Force resolution/controllability of the device as afforded by the actuation system. Score 0: low; Score 1: reasonable; Score 2: high.

C4.5 - Passive compliance of the device as afforded by the actuation system, in relation to the device requirements. Depending on device type and application, compliance may be a desirable property. For example, in artificial hands that operate in unstructured environments, the active compliance (through control) and/or passive compliance (through mechanical elements) contribute to several important objectives such as grasp adaptability, grasp stability, and safety during interaction. This criterion takes into consideration the degree of passive compliance in the actuation system, in relation to the requirements of the device. (Active compliance considerations are included with controllability in C4.3 and C4.4.) Score 0: the approach to passive compliance does not support the device requirements; Score 1: some degree of passive compliance is reported or is apparent, but is not optimized to the device requirements; Score 2: the degree of passive compliance matches the device requirements.

C4.6 - Ranges of motion of the device joints in relation to the device expectations. In the case of anthropomorphic hands, the ranges of joint motion attributed to the human finger joints [64], guide the evaluation. Score 0: all or a considerable number do not fulfil the expectations to a significant degree; Score 1: all fall slightly short of the expectations, or only a few do not fulfil the expectations; Score 2: all fulfil or exceed the expectations.

C4.7 - Capability of an actuator to simultaneously produce independently controllable motions in non-coplanar planes as required by the device type. Some device types may require independently controllable motions in non-coplanar planes. For example, the base 
of each finger in an anthropomorphic hand may have one DOF for flexion-extension movements and one DOF for adduction-abduction. Normally, two independent actuators provide these motions. However, multi-DOF actuators capable of producing these motions while using only one 'motor' have been developed for several applications. Examples include the multi-DOF ultrasonic motor in the base joint of a prototype robot finger [65], and a similar one that controls the tip of a surgical forceps [66]. This criterion considers such solutions positively. There are also transmission systems such as the Geneva-wheel mechanism implemented in [8] that permit one actuator to produce controllable motions in non-coplanar planes. The Geneva-wheel mechanism can produce controllable motion in two non-coplanar planes intermittently and in a cyclic manner, i.e. it is possible to switch from motion in one plane to the other only at a specific position in the movement cycle, rather than at any position. Although such mechanisms are not actuators, their capabilities are included for consideration here. Score 0: not capable; Score 1: capable with one actuator, but not simultaneously in several planes; Score 2: capable, or not applicable to the device type.

C4.8 - Effects of the electrical/fluidic lines of the actuation system on the motions of the endpart. Score 0: significant effects reported or apparent; Score 1: some effects reported or apparent; Score 2: no or minimal effects reported or apparent.

C4.9 - Energy Consumption of the actuation system in relation to the end-part expectations. Score 0: reported or expected to be relatively wasteful; Score 1: some measures that reduce the consumption were reported or are apparent; Score 2: a significant performance was reported, or considerable measures that reduce the consumption were taken.

C4.10 - Degree of suitability of the actuation system for the device environment. The criterion takes into consideration the level of general suitability of the actuation system and related packaging in the intended environment of deployment. The safety of actuators used in devices such as prosthetic hands, and domestic oriented humanoid robots/devices intended to interact with human beings and other forms of life may be addressed under this aspect as well. Additionally, the evaluation is attentive to the efforts carried out in devices that cater for special environments such as outer space. Score 0: has some notable deficiencies in suitability; Score 1: has some minor deficiencies in suitability; Score 2: extremely suitable.

C4.11 - Modification flexibility provided by the actuation system in relation to the end-part expectations. This criterion considers the level of flexibility for modification permitted by the actuation system (typically through modularity) at the finger configuration level and/or in the actuation architecture. Score 0 : the actuation system does not provide flexibility for modification; Score 1: the actuation system permits some degree of flexibility; Score 2: the actuation system permits significant flexibility, or not applicable to device type. 
C4.12 - Noise level of the actuation system with respect to the end-part expectations. Score 0 : a significant noise level reported or the possibility of existence is apparent; Score 1: reported or expected to be somewhat noisy; Score 2: reported or expected to generate low or acceptable noise levels.

C4.13 - Effect of the form of the actuation system on the end-part function. The final form, including the aspect ratio of the mounted actuators and transmission mechanisms might affect the device function. For instance in SMA actuator wires as well as in linear PAM actuators, the amount of contraction is a function of the original length. Hence, even if used in conjunction with a stroke amplifying mechanism, a specific length of actuator is required to achieve the desired range of motion. For this reason in Hand 34 ([51]) for example, the range of motion of some joints is limited because of restrictions in the length of SMA that the hand could accommodate. Considering Hand 11 ([29]), which makes use of ultrasonic motors and pulley-tendon transmission stages, the construction of the actuation system in the modular fingers resulted in a flat (and rigid) palm, which is not optimal during power grasps. Score 0: significant effect reported or apparent; Score 1: some effect reported or apparent; Score 2: no effect reported or apparent.

C4.14 - Cost of the actuators in relation to the average actuator cost for the device type in the given power range. Score 0: high - more than twice the estimated average; Score 1: average - between half and twice the estimated average; Score 2: low - less than half the estimated average.

\section{The Quantification of AD in Five Specific Test Cases}

In order to demonstrate the approach, five of the reviewed hand projects were selected as case studies for the quantification of $A D$. The selections were carried out on the basis of (i) having a representative set of different types of actuation approaches and of actuators in the sample; and (ii) sufficient detail on the projects being available to enable the accurate evaluation of all or most of the criteria. The selected hand projects (refer to Table 1 ) were:

Hand 6 The DLR/HIT Hand II, a human-scale, modular, five-fingered hand exhibiting 15 DOF, and with an integrated actuation system based on brushless DC motors.

Hand 11 A human-scale, modular, five-fingered hand from Keio University, exhibiting 20 DOF and with an integrated actuation system based on ultrasonic motors.

Hand 12 A miniature, five-fingered hand also from Keio University, exhibiting 20 DOF and driven by SMA actuators located in a forearm.

Hand 19 A human-scale, modular, five-fingered hand from the Karlsruhe Research Centre (FZK), exhibiting 8 active joints driven through 2 DOF, and with an integrated actuation system based on flexible fluidic actuators. 
Hand 36 The Utah/MIT Dextrous Hand, a four-fingered hand, somewhat larger than the human counterpart, exhibiting 16 DOF and driven by custom-made pneumatic cylinders located remotely.

A summary of the results obtained is given in Table 3. For information purposes, the table compares the results assigned to these five artificial hands to the corresponding results assigned to the human hand. The column labelled $H 1$ refers to the result obtained through strict adherence to all of the criteria evaluation boundaries. The column labelled $H 2$ refers to a corrected result (i.e. enhanced score) for the human hand where compensatory factors that are unique to the natural organ (and that are presently unattainable technologically) are taken into consideration. This issue of compensation for low individual $A D$ criterion scores is discussed further at the end of section 8.2. It is noted that the human hand does not achieve a full score when tested using the AD criteria compiled in this work, since there are several aspects, relevant to $A D$, in which an artificial device (in this case a robotic or prosthetic hand) can surpass its natural counterpart.

\begin{tabular}{|c|c|c|c|c|c|c|c|c|c|c|c|c|c|c|c|c|c|}
\hline \multirow[t]{2}{*}{ Crit. } & \multirow[t]{2}{*}{ Short Description } & \multicolumn{5}{|c|}{ Artificial hands } & \multicolumn{2}{|c|}{$\begin{array}{c}\text { Human } \\
\text { hand }\end{array}$} & \multirow[t]{2}{*}{ Crit. } & \multirow[t]{2}{*}{ Short Description } & \multicolumn{5}{|c|}{ Artificial hands } & \multicolumn{2}{|c|}{$\begin{array}{c}\text { Human } \\
\text { hand }\end{array}$} \\
\hline & & 6 & 11 & 12 & 19 & 36 & H1 & $\mathrm{H2}$ & & & 6 & 11 & 12 & 19 & 36 & \begin{tabular}{|l|l|} 
H1 \\
\end{tabular} & $\mathrm{H} 2$ \\
\hline$\overline{\mathrm{C} 1.1}$ & Specific power, A & 1 & 1 & 0 & 2 & 1 & 1 & 1 & C3.1 & Mass AS/D & 2 & 1 & 0 & 2 & 0 & 1 & 1 \\
\hline $\mathrm{C} 1.2$ & Volumetric power, $\mathrm{A}$ & 0 & 0 & 0 & 2 & 1 & 0 & 0 & $\mathrm{C} 3.2$ & Volume AS/D & 1 & 1 & 0 & 2 & 0 & 1 & 1 \\
\hline $\mathrm{C} 1.3$ & Maximum strain, $\mathrm{A}$ & 2 & 2 & 0 & 2 & 1 & 1 & 1 & $\mathrm{C} 3.3$ & Output force / AS mass & 1 & 1 & 0 & 2 & 1 & 1 & 1 \\
\hline C1.4 & Force constancy/linearity, A & 2 & 2 & 0 & 1 & 2 & 0 & $2^{*}$ & C3.4 & Output force / AS volume & 1 & \begin{tabular}{|l|}
1 \\
\end{tabular} & 0 & 2 & 0 & 0 & 0 \\
\hline C1.5 & Hysteresis, A & 2 & 2 & 0 & 1 & 1 & 1 & 2* & $\mathrm{C3.5}$ & Output force / internal force & 1 & 1 & 1 & 2 & 1 & 1 & 1 \\
\hline C1.6 & Efficiency, A & 1 & 0 & 0 & 2 & 1 & 0 & 0 & C3.6 & AS contr. to res/acc/rep & 2 & 2 & 1 & 0 & 1 & 1 & $2^{*}$ \\
\hline $\mathrm{C} 1.7$ & Complexity, A & 1 & 0 & 2 & 2 & 0 & 2 & 2 & $\mathrm{C3.7}$ & Aesthetics, effect of AS on D & 1 & 1 & 2 & 2 & 0 & 2 & 2 \\
\hline C1.8 & Need for transmission & 0 & 0 & 0 & 1 & 0 & 0 & 0 & $A D$ & Category 3 score & 9 & 8 & 4 & 12 & 3 & 7 & 8 \\
\hline C1.9 & Complexity, $T$ & 0 & 2 & 2 & 2 & 0 & 2 & 2 & & & & & & & & & \\
\hline C1.10 & Route, $T$ & 1 & 1 & 1 & 2 & 0 & 0 & 2* & $\mathrm{C} 4.1$ & Joint speeds, D & 1 & 1 & 1 & 0 & 2 & 2 & 2 \\
\hline C1.11 & Interference, $\mathrm{T}$ & 2 & 1 & 1 & 2 & 0 & 1 & $2^{*}$ & $C 4.2$ & Time constant, D & 2 & 1 & 1 & 0 & 2 & 2 & 2 \\
\hline C1.12 & Inertia, $T$ & 1 & 2 & 2 & 1 & 2 & 2 & 2 & $C 4.3$ & Position resolution, $\mathrm{D}$ & 2 & 2 & 1 & 1 & 1 & 1 & 1 \\
\hline C1.13 & Mass A/AS & 0 & 1 & 0 & 0 & 0 & 2 & 2 & C4.4 & Force resolution, $\mathrm{D}$ & 2 & 2 & 1 & 1 & 1 & 1 & 1 \\
\hline C1.14 & Volume A/AS & 0 & 0 & 0 & 1 & 0 & 2 & 2 & $\mathrm{C} 4.5$ & Passive compliance, D & 1 & 2 & 0 & 2 & 2 & 1 & $2^{*}$ \\
\hline C1.15 & Internal resistance, AS & 1 & 1 & 1 & 1 & 1 & 2 & 2 & $C 4.6$ & Ranges of motion, $\mathrm{D}$ & 1 & 2 & 1 & 1 & 2 & 2 & 2 \\
\hline C1.16 & Energy saving features, AS & 0 & 2 & 0 & 1 & 0 & 1 & 1 & C4.7 & Multi-plane capability, A & 0 & 0 & 0 & 0 & 0 & 0 & $2^{*}$ \\
\hline C1.17 & Identicality, A, T & 2 & 2 & 2 & 2 & 2 & 1 & 2* & C4.8 & Lines, AS wrt motions, D & 2 & 0 & 0 & 1 & 2 & 2 & 2 \\
\hline C1.18 & Modularity/maintenance, AS & 1 & 1 & 1 & 2 & 2 & 0 & $1 * *$ & $C 4.9$ & Energy consumption, AS & 1 & 1 & 1 & 1 & 0 & 2 & 2 \\
\hline C1.19 & Reliability, AS & 2 & 0 & 0 & 2 & 2 & 2 & 2 & C4.10 & Suitability for environment, AS & 2 & 1 & 0 & 2 & 2 & 2 & 2 \\
\hline $\mathrm{C} 1.20$ & Smoothness, AS & 2 & 0 & 1 & 2 & 2 & 2 & 2 & C4.11 & Flexibility, AS & 1 & 2 & 2 & 1 & 2 & 0 & 0 \\
\hline \multirow[t]{2}{*}{$A D$} & Category 1 score & 21 & 20 & 13 & 31 & 18 & 22 & 30 & $C 4.12$ & Noise level, AS & 1 & 2 & 2 & 2 & 0 & 2 & 2 \\
\hline & & & & & & & & & C4.13 & Function, D wrt form, AS & 2 & 1 & 1 & 1 & 1 & 2 & 2 \\
\hline C2.1 & Use of same power supply & 2 & 2 & 2 & 1 & 0 & 2 & 2 & C4.14 & Cost, A & 1 & 1 & 2 & 2 & 0 & 2 & 2 \\
\hline $\mathrm{C} 2.2$ & Need for internal sensors & 0 & 0 & 0 & 0 & 0 & 1 & 1 & $A D$ & Category 4 score & 19 & 18 & 13 & 15 & 17 & 21 & 24 \\
\hline $\mathrm{C} 2.3$ & Adaptable passive joints & 0 & 2 & 2 & 1 & 2 & 0 & 0 & & & & & & & & & \\
\hline $\mathrm{C} 2.4$ & AS mass / D DOF & 2 & 2 & 0 & 1 & 0 & 2 & 2 & & & & & & & & & \\
\hline $\mathrm{C} 2.5$ & AS volume / D DOF & 2 & 2 & 1 & 2 & 0 & 2 & 2 & & & & & & & & & \\
\hline $\mathrm{C} 2.6$ & AS mass / $D$ joint & 2 & 2 & 0 & 2 & 0 & 2 & 2 & & & & & & & & & \\
\hline $\mathrm{C} 2.7$ & AS volume / D joint & 2 & 2 & 0 & 2 & 0 & 2 & 2 & & & & & & & & & \\
\hline AD & Category 2 score & 10 & 12 & 5 & 9 & 2 & 11 & 11 & $\overline{A D}$ & Total Score & 59 & 58 & 35 & 67 & 40 & \begin{tabular}{|l|}
61 \\
\end{tabular} & 73 \\
\hline
\end{tabular}

(Abbreviations: A - Actuators; T - Transmission system; AS - Actuation system; D - Device; wrt - with respect to)

* hand / control system optimized through evolution

** limited self-healing capability

Table 3.

$A D$ evaluation results 


\section{Discussion and Implications of Results}

\subsection{Brief Analysis of the Demonstration Results}

The evaluation and comparison exercise summarized in section 7 was carried out for demonstration purposes, and the results in Table 3 should be interpreted as a measure of the extent to which the various issues relating to actuation are addressed (by the design approach to actuation, and inherently by the selected technologies), rather than as a statement on which of the five artificial specimens is the best hand. Hand 19, for example, which obtained the highest overall AD score, has the lowest number of DOF and the slowest joint speeds among the five artificial hands, and would therefore be unsuitable for many applications. The results obtained through the AD evaluation can be taken to indicate however that if certain identified shortcomings can be overcome and/or improvements made, the design approach to actuation taken for Hand 19, including the choice of actuator, has a high potential for use in artificial hands.

Hand 19 scores high in Category 1, due mainly to the ability of its actuation system to work without a transmission system, and in Category 3, due mainly to the light and compact physical properties of its actuation system in relation to the properties and force capabilities of the device. Hands 6 and 11 score high in Category 2, mainly due to their large number of joints and DOF in relation to the actuation system mass and size. Hands 6,11 , and 36 score high in Category 4, due to the high extent to which their actuation systems result in the meeting of the general expectations of a dexterous hand.

Hand 12 scores low in all categories, due to the relatively disadvantageous physical properties of the implemented actuators, and the low extent to which its actuation system satisfies the general expectations of a dexterous hand. Hand 36 scores low in categories 2 and 3 , mainly due to the relatively large size and mass of the actuation system.

\subsection{Implications for Device Design}

The implications of this exercise on the design of artificial hands are significant, but they should be extracted with care. The AD criteria do not represent a strict recipe for the design of an actuation system, since in most practical situations certain aspects may need to be sacrificed in order to attain large benefits in other aspects of the design. Rather, the AD criteria are intended to serve as a checklist for the designer of the device, and are (i) useful triggers to urge the designer to consider radical issues even during the early design process (e.g. is there any way to obtain all the required device properties using an actuation system that does not require a transmission?); (ii) general guidelines to help the designer throughout the entire design process (e.g. can the passive joints be designed to have modifiable motion ratios with respect to the active joints to which they are coupled?); and (iii) useful pointers to optimize the design even during the later stages (e.g. can the actuation system be fine tuned to minimize its impact on the aesthetics of the device?). An 
ideal actuation approach / system (with respect to the criteria and their scoring boundaries as set out in this work) would indeed be one that returns the maximum AD score of 96, and therefore this can be considered an ultimate objective for designers of artificial hands.

The unique viewpoint that is afforded through the multifactorial and systematic evaluation of actuation dexterity also serves to highlight certain general considerations that might otherwise remain more obscure. Thus for example, the latent potential associated with flexible fluidic actuators, indicated in section 8.1 above, is underscored through this evaluation approach. At the same time, the study highlights the critical, practical shortcomings of SMAs, sometimes considered as belonging to the set of highly promising non-conventional actuators of the future for certain MDOF applications. An implicit conclusion that can be drawn from this study is that in order to optimize the AD score, it is likely that actuators (as well as all other elements of the actuation system) will need to be custom designed for the specific MDOF device to which they will be applied. In this way, one can achieve optimum synergy between the actuation approach / system and the device that it is meant to serve, and this would also avoid inaccurate conclusions such as that highlighted in [6] and discussed in section 2.1 above.

Those criteria for which the result for the human hand in Table 3 was corrected to a higher score also provide some enlightening input to this discussion. Thus for example although the human muscle, as an actuator, has a force output that varies in a non-linear way with position, and even exhibits some degree of hysteresis, the human body enjoys the benefits of millions of years of evolution, and its motor control system has found ways to eliminate the normally detrimental effects of these theoretical shortcomings in a seamless manner. Thus, even in the design of artificial hands, it may be possible to compensate for a specific low criterion score, obtained through the strict application of the scoring boundaries, through the use of a radical technological or analytical/computational breakthrough that nullifies the detrimental effects of the low score without incurring any other penalties (such as added complexity).

\subsection{Applicability of the AD evaluation criteria to a different device type}

The constituent elements, properties and manifestations of the universal elements for MIS devices are very similar to those for artificial hands given in Fig. 5. Slight differences exist for the contact interface, where manifestations could include tips, sharp edges, holding surfaces or other tools depending on the surgical procedure; and for the hardware interfaces, which could include specially designed interfaces necessary to enable the (ideally quick) separation and reconnection of specific modules for sterilization purposes. The constituents of the universal elements of the actuation system for MIS devices are identical to those for artificial hands shown in Fig. 6.

A review of the forty-eight $A D$ evaluation criteria with respect to MIS devices reveals that all of them can be applied directly to these devices, and also that it is quite likely that in most if 
not all cases it would be possible to apply directly the same score boundaries derived for artificial hands, due to the universal and normalized nature of the criteria, and due to the fact that both of these device types are being evaluated with respect to the same baseline technological capability that is available today. In particular, this common technological baseline permits the retention of the scoring boundaries even in the cases where these have been derived following non-dimensionalization with respect to the human hand. This portability of the AD evaluation method between different device types is one of its main distinctive features.

\section{Conclusion}

The basic formulation presented in this work is intended to serve as a demonstration of the $A D$ concept and of its application. Further improvements or variants of this approach may consist of the evaluation criteria being extended or changed, and/or of the scoring boundaries of the criteria being revised. At some cost in objectivity and portability, other variants of the approach may involve an increased number of scoring options per criterion, and/or relative weightings being assigned to the criteria. In situations where the approach is to be used as the primary actuation system selection tool, such weightings would be customized for the specific device under consideration as part of the product design specification of the device.

The detailed study and evaluation of the numerous issues that contribute to dexterous actuation, and the hard quantification of this attribute through approaches such as the $A D$ measure presented in this work, serve to highlight the many factors that influence or are influenced by the actuation system of an MDOF device that has space and weight constraints. All of these factors should be taken into consideration during the design and development of such devices. Just as importantly, this evaluation approach may help the designer to understand better and to rationalize the various trade-offs that can be made, and their significance, during the design of such systems. Finally, the absolute AD score of a device serves to give a distinctive indication of how close the device has come to achieving and incorporating an ideal total actuation solution.

\section{Acknowledgements}

This work was partially funded by the University of Malta through Internal Research Grants, numbers $73-528$ and 31-353.

\section{References}

[1] C. Melchiorri, M. Kaneko, Robot hands, in Handbook of robotics, B. Siciliano and $O$. Khatib, Eds., Springer Verlag, Berlin-Heidelberg, Germany (2008), pp. 345-360.

[2] V.R. Kode, M.C. Cavusoglu, M.T. Azar, Design and Characterization of a Novel Hybrid Actuator using Shape Memory Alloy and D.C Motor for Minimally Invasive Surgery 
Applications, Proceedings of the IEEE International Conference on Mechatronics and Automation (2005) 416-420.

[3] J.E. Huber, N.A. Fleck, M.F. Ashby, The selection of mechanical actuators based on performance indices, Proceedings of the Royal Society of London A (1997) 453, 21852205.

[4] M. Zupan, M.F. Ashby, N.A. Fleck, Actuator classification and selection - the development of a database, Advanced Engineering Materials 4(12) (2002) 933-940.

[5] A.D. Poole, J.D. Booker, Design methodology and case studies in actuator selection, Mechanism and Machine Theory 46 (2011) 647-661.

[6] D.H. Plettenburg, Pneumatic actuators: a comparison of energy-to-mass ratios, Proceedings of the IEEE International Conference on Rehabilitation Robotics (2005) 545-549.

[7] V.O. Del Cura, F.L. Cunha, M.L. Aguiar, A. Cliquet, Jr, Study of the different types of actuators and mechanisms for upper limb prostheses, Artificial Organs 27 (2003) (6) 507-516.

[8] J.L. Pons, E. Rocon, R. Ceres, D. Reynaerts, B. Saro, S. Levin, W. van Moorleghem, The MANUS-HAND dextrous robotics upper limb prosthesis: mechanical and manipulation aspects, Autonomous Robots 16 (2004) 143-163.

[9] P. Dario, RobotCub: development of a cognitive humanoid cub, Deliverable no. 7.2: Analysis and pre-selection of the sensor and actuation technologies (2005), The RobotCub Consortium, European Commission FP6 Project IST-004370.

[10] F.C. Park, R.W. Brockett, Kinematic dexterity of robotic mechanisms, International Journal of Robotics Research, 13 (1994) (1) 1-15.

[11] J. Angeles, F.C. Park, Performance evaluation and design criteria, in Handbook of robotics, B. Siciliano and O. Khatib, Eds., Springer Verlag, Berlin-Heidelberg, Germany (2008), pp. 229-243.

[12] C.M. Gosselin, J. Angeles, A new performance index for the kinematic optimization of robotic manipulators, ASME Trends and Developments in Mechanisms, Machines and Robotics, DE-Vol. 15-3 (1988) 441-447.

[13] L. Biagiotti, F. Lotti, C. Melchiorri, G. Vassura, How far is the human hand? A review on anthropomorphic robotic end-effectors, DEIS - DIEM Internal Report (2004), University of Bologna.

[14] L. Lu, C. Cai, A.H. Soni, Object oriented dexterity analysis and design application for spatial robot hands, ASME Design Technology Conference (1989), Kissimmee, FL.

[15] R.H. Sturges, A quantification of machine dexterity applied to an assembly task, The International Journal of Robotics Research 9(3) (1990) 49-62.

[16] S. Awtar, T.T. Trutna, J.M. Neilsen, R. Abani, J. Geiger, FlexDex: a minimally invasive surgical tool with enhanced dexterity and intuitive control, Journal of Medical Devices 4, 035003 (2010). 
[17] M. C. Carrozza, C. Suppo, F. Sebastiani, B. Massa, F. Vecchi, R. Lazzarini, M.R. Cutkosky, P. Dario, The SPRING hand: development of a self-adaptive prosthesis for restoring natural grasping, Autonomous Robots 16 (2004) 125-141.

[18] I. Kawabuchi, A designing of humanoid robot hands in endoskeleton and exoskeleton styles, in Humanoid robots, new developments, A. Carlos de Pina Filho, Ed., I-Tech, Vienna, Austria (2007) 401-426.

[19] M. C. Carrozza, B. Massa, S. Micera, R. Lazzarini, M. Zecca, P. Dario, The development of a novel prosthetic hand-ongoing research and preliminary results, IEEE/ASME Transactions on Mechatronics, 7 (2002) (2) 108-114.

[20] W. Townsend, The BarrettHand grasper - programmably flexible part handling and assembly, Industrial Robot: An International Journal 27 (2000) 181-188.

[21] J. Butterfass, G. Hirzinger, S. Knoch, H. Liu, DLR's multisensory articulated hand. Part I: Hard- and software architecture, Proceedings of the IEEE International Conference on Robotics and Automation (1998) 2081-2086.

[22] J. Butterfass, M. Grebenstein, H. Liu, G. Hirzinger, DLR-hand II: Next generation of a dextrous robot hand, Proceedings of the IEEE International Conference on Robotics and Automation (2001) 109-114.

[23] H. Liu, P. Meusel, G. Hirzinger, M. Jin, Y. Liu, Z. Xie, The modular multisensory DLR-HIThand: hardware and software architecture, IEEE/ASME Transactions on Mechatronics 13 (2008) 461-469.

[24] H. Liu, K. Wu, P. Meusel, N. Seitz, G. Hirzinger, M.H. Jin, Y.W. Liu, S.W.Fan, T. Lan, Z.P.Chen, Multisensory five-finger dexterous hand: the DLR/HIT hand II, IEEE/RSJ International Conference on Intelligent Robots and Systems (2008) 3692-3697.

[25] H. Kawasaki, T. Komatsu, K. Uchiyama, Dexterous anthropomorphic robot hand with distributed tactile sensor: Gifu hand II, IEEE/ASME Transactions on Mechatronics 7 (2002) 296-303.

[26] T. Mouri, H. Kawasaki, K. Yoshikawa, J. Takai, S. Ito, Anthropomorphic robot hand: Gifu hand III, Proceedings of the International Conference on Control, Automation and Systems (2002) 1288-1293.

[27] H. Kawasaki, T. Mouri, S. Ito, Toward next stage of kinematic humanoid hand, Proceedings of World Automation Congress, 10th International Symposium on Robotics with Applications (2004) 129-134.

[28] T. Mouri, H. Kawasaki, K. Umebayashi, Developments of new anthropomorphic robot hand and its master slave system, Proceedings of the IEEE/RSJ International Conference on Intelligent Robots and Systems (2005) 3474-3479.

[29] I. Yamano, T. Maeno, Five-fingered robot hand using ultrasonic motors and elastic elements, Proceedings of the IEEE International Conference on Robotics and Automation (2005) 2684-2689.

[30] T. Maeno, T. Hino, Miniature five-fingered robot hand driven by shape memory alloy actuators, Proceedings of the IASTED International Conference on Robotics and Applications (2006) 174-179. 
[31] K.-J. Cho, J. Rosemarin, H. Asada, Design of vast DOF artificial muscle actuators with a cellular array structure and its application to a five-fingered robotic hand, Proceedings of the IEEE International Conference on Robotics and Automation (2006) 2214-2219.

[32] J. Ueda, Y. Ishida, M. Kondo, T. Ogasawara, Development of the NAIST-hand with vision-based tactile fingertip sensor, Proceedings of the IEEE International Conference on Robotics and Automation (2005) 2343-2348.

[33] C.S. Lovchik, H.A. Aldridge, M.A. Diftler, Design of the NASA Robonaut hand, Proceedings of the ASME Dynamics and Control Division (1999) 813-830.

[34] K. Kaneko, K. Harada, F. Kanehiro, Development of multi-fingered hand for life-size humanoid robots, IEEE International Conference on Robotics and Automation (2007) 913-920.

[35] D. Sasaki, T. Noritsugu, M. Takaiwa, Development of pneumatic soft robot hand for human friendly robot, Journal of Robotics and Mechatronics 15 (2003) 164-171.

[36] S. Schulz, C. Pylatiuk, G. Bretthauer, A new ultralight anthropomorphic hand, Proceedings of the IEEE International Conference on Robotics and Automation (2001) 2437-2441.

[37] A. Kargov, T. Asfour, C. Pylatiuk, R. Oberle, H. Klosek, S. Schulz, K. Regenstein, G. Bretthauer, R. Dillmann, Development of an anthropomorphic hand for a mobile assistive robot, IEEE International Conference on Rehabilitation Robotics: Frontiers of the Human-Machine Interface (2005) 182-186.

[38] S. Schulz, Ch. Pylatiuk, A. Kargov, R. Oberle, H. Klosek, T. Werner, R. Rößler, Development of a Multifunctional Cosmetic Hand Prosthesis, Orthopädie-Technik 57 (2006) 627-632.

[39] M.C. Carrozza, G. Cappiello, S. Micera, B.B. Edin, L. Beccai, C. Cipriani, Design of a cybernetic hand for perception and action, Biological Cybernetics 95 (2006) 629-644.

[40] Shadow Robot Company Ltd., Shadow dexterous hand C5 technical specification release: May 14, 2008.

[41] Shadow Robot Company Ltd., Shadow dexterous hand C6M technical specification release: August 15, 2009.

[42] F. Lotti, P. Tiezzi, G. Vassura, L. Biagiotti, G. Palli, C. Melchiorri, Development of UB hand 3: early results, Proceedings of the IEEE International Conference on Robotics and Automation (2005) 4488-4493.

[43] N.E.N. Rodríguez, G. Carbone, E. Ottaviano, M. Ceccarelli, An experimental validation of a three-fingered hand with 1 DOF anthropomorphic fingers, Proceedings of the International Conference on Intelligent Manipulation and Grasping (2004) 285-290.

[44] A. Caffaz, G. Casalino, G. Cannata, G. Panin, E. Massucco, The DIST-hand, an anthropomorphic, fully sensorized dexterous gripper, IEEE-RAS International Conference on Humanoid Robotics, Cambridge, MA, 2000.

[45] M.A. Saliba, M. Axiak, Design of a Compact, Dexterous Robot Hand with Remotely Located Actuators and Sensors, Proceedings of the IEEE Mediterranean Conference on Control and Automation, Athens, Greece, 2007. 
[46] C.M. Light, P.H. Chappell, Development of a lightweight and adaptable multiple-axis hand prosthesis, Medical Engineering \& Physics 22 (2000) 679-684.

[47] Y.K. Lee, I. Shimoyama, A skeletal framework artificial hand actuated by pneumatic artificial muscles, Proceedings of the IEEE International Conference on Robotics and Automation (1999) 926-931.

[48] A.H. Arieta, R. Katoh, H. Yokoi, Y. Wenwei, Development of a multi-DOF electromyography prosthetic system using the adaptive joint mechanism, Applied Bionics and Biomechanics 3 (2006) 101-112.

[49] H. Kaminaga, T. Yamamoto, J. Ono, Y. Nakamura, Backdrivable miniature hydrostatic transmission for actuation of anthropomorphic robot hands, IEEE-RAS International Conference on Humanoid Robots (2007) 36-41.

[50] N. Dechev, W.L. Cleghorn, S. Naumann, Multi-segmented finger design of an experimental prosthetic hand, Proceedings of the Sixth National Applied Mechanisms and Robotics Conference, Cincinnati, OH, 1999.

[51] A.D. Price, A. Jnifene, H.E. Naguib, Design and control of a shape memory alloy based dexterous robot hand, Smart Materials and Structures 16 (2007) 1401-1414.

[52] K. Hoshino, I. Kawabuchi, Pinching at fingertips for humanoid robot hand, Journal of Robotics and Mechatronics 17 (2005) 655-663.

[53] S.C. Jacobsen, E.K. Iversen, D.F. Knutti, R.T. Johnson, and K.B. Biggers, Design of the UTAH/M.I.T. Dextrous hand, Proceedings of the IEEE International Conference on Robotics and Automation (1986) 1520-1532.

[54] C. Ellul, M.A. Saliba, Comparative analysis of artificial hands: the need for reporting and test standards, Proceedings of the IEEE International Conference on Applied Bionics and Biomechanics, Venice, Italy, 2010.

[55] J.M. Hollerbach, I.W. Hunter, J. Ballantyne, A comparative analysis of actuator technologies for robotics, The robotics review 2, O. Khatib, et al., Eds., MIT Press, 1992, pp. 299-342.

[56] J. Peirs, D. Reynaerts, H. Van Brussel, A retrospective evaluation of SMA microactuation, Proceedings of the International Conference on New Actuators (2002), Bremen, Germany, pp. 77-80.

[57] I.W. Hunter, S. Lafontaine, A comparison of muscle with artificial actuators, IEEE Solid State Sensor and Actuator Workshop, $5^{\text {th }}$ Technical Digest (1992) 178-185.

[58] H.Z. Tan, M.A. Srinivasan, B. Eberman, B. Cheng, Human factors for the design of forcereflecting haptic interfaces. Proceedings of the International Symposium on Haptic Interfaces for Virtual Environment and Teleoperator Systems, ASME Dynamic Systems and Control Division 55 (1994) (1) 353-359.

[59] C.E. Clauser, J.T. McConville, J.W. Young, Weight, volume, and center of mass of segments of the human body, US Department of Commerce, National Technical Information Service (1969).

[60] W.G. Darling, K.J. Cole, Muscle activation patterns and kinetics of human index finger movements, Journal of Neurophysiology, 63 (1990) 1098-1108. 
[61] C.J. Hasser, Force reflecting anthropomorphic handmaster requirements, Proceedings of the ASME Dynamic Systems and Control Division DSC-Vol. 57-2 (1995) 663-674.

[62] B.R. Umberger, K.G.M. Gerritsen, P.E. Martin, A model of human muscle energy expenditure, Computer Methods in Biomechanics and Biomedical Engineering 6 (2003) (2) 99-111.

[63] E. de Vlugt, A.C. Schouten, F.C.T. van der Helm, Quantification of intrinsic and reflexive properties during multijoint arm posture, Journal of Neuroscience Methods 155 (2006) 328-349.

[64] J. Lin, Y. Wu, T.S. Huang, Modeling the constraints of human hand motion. IEEE Workshop on Human Motion (2000), Los Alamitos, CA, pp. 121-126.

[65] X. Zhang, Y. Gouda, D. Koyama, K. Nakamura, S. Ueha, A basic design of robot finger joint using multi-degree-of-freedom ultrasonic motor, Acoustical Science and Technology 29 (2008) 235-237.

[66] S. Park, K. Takemura, T. Maeno, Study on multi-DOF ultrasonic actuator for laparoscopic instrument, JSME International Journal Series C 47 (2004) 574-581. 


\section{Figure Captions}

Fig. 1. Expected roles of the DAAP in the development process of a MDOF device

Fig. 2. Fields of application of the reviewed projects

Fig. 3. Breakdown of the main features of the reviewed projects

Fig. 4. Coupling mechanisms for the passive joints

Fig. 5. Universal elements of a MDOF device

Fig. 6. Universal elements of the actuation system 